\title{
Involvement of a Phosphorylation-Mediated Pathway to Regulate the Function of NSPL1 in Exercise
}

\author{
Takaaki IKEMOTO ${ }^{1) *}$, Masaaki SUZUKI ${ }^{2)}$ and Hirotaka ONOE ${ }^{1)}$ \\ ${ }^{1)}$ Functional Probe Research Laboratory, RIKEN Center for Molecular Imaging Science, Kobe 650-0047 and \\ ${ }^{2}$ Molecular Imaging Medicinal Chemistry Laboratory, RIKEN Center for Molecular Imaging Science, Kobe 650-0047, Japan
}

(Received 8 December 2010/Accepted 5 January 2011/Published online in J-STAGE 19 January 2011)

\begin{abstract}
Skeletal-type neuroendocrine-specific protein like 1 (sk-NSPL1) has been demonstrated to be physiologically important in regulating the membrane translocation of glucose transporter 4 (GLUT4) in skeletal muscles. We investigated the levels of phosphorylation in proteins that are thought to be involved in exercise in wild-type and sk-NSPL1-deficient muscles with specific antibodies and phosphate-metal affinity chromatography resin (p-resin). In both normal skeletal muscle and sk-NSPL1-deficient muscle, adenosine monophosphate (AMP)-dependent kinase (AMPK) and acetyl-CoA carboxylase (ACC) were phosphorylated and adsorbed onto p-resin at high levels after exercise. On the other hand, the effect of 5-aminoimidazole-4-carboxyamide ribonucleoside (AICAR), which is an activator of AMPK, in blood glucose was greatly diminished in mutant mice. P-resin adsorbed sk-NSPL1 in the membrane fraction from wildtype muscle after exercise and AICAR administration. Isolated sk-NSPL1 from wild-type also had increased adsorption onto p-resin after treatment with $\mathrm{Ca}^{2+}$ and adenosine triphosphate (ATP). After long-term incubation of sk-NSPL1-containing membrane without ATP, sk-NSPL1 adsorption onto anion-exchange resin was drastically reduced. These results suggest that the function of sk-NSPL1 is regulated by a $\left[\mathrm{Ca}^{2+}\right]_{\mathrm{i}}$ - and AMPK-mediated pathway under exercise, and support the hypothesis that sk-NSPL1 is an important factor in the downstream of the exercise-dependent pathway in GLUT4 translocation.

KEY WORDS: exercise, GLUT4 translocation, insulin, phosphorylation, skeletal muscle.
\end{abstract}

J. Vet. Med. Sci. 73(6): 733-738, 2011

Glucose uptake in skeletal muscles, which is regulated by membrane translocation of glucose transporter 4 (GLUT4) from internal stores to the cell membrane, can change blood glucose levels in the whole body [7, 14]. Therefore, resolving the molecular mechanisms that underlie this function is important. We previously reported that skeletal-type neuroendocrine-specific protein-like 1 (sk-NSPL1) plays an important role in GLUT4 translocation induced by contraction/exercise in skeletal muscles [9]. However, the precise mechanism by which sk-NSPL1 works has not been elucidated.

In the present study, we examined the level of phosphorylation of adenosine monophosphate (AMP)-dependent kinase (AMPK) in sk-NSPL1-deficient muscle, which is affected by changes in AMP/adenosine triphosphate (ATP) in cells under muscle contraction, and the effect of 5-aminoimidazole-4-carboxyamide ribonucleoside (AICAR) on blood glucose, which can activate AMPK $[12,17]$. Our results suggest that sk-NSPL1 might be involved downstream of the AMPK-mediated pathway in GLUT4 translocation. We also investigated whether isolated sk-NSPL1 adsorbed on to phosphate-metal affinity chromatography resin (p-resin) in response to contraction or by elevation of $\left[\mathrm{Ca}^{2+}\right]_{\mathrm{i}}$ within physiological levels. Our data suggest that a phosphorylation-mediated change in function of sk-NSPL1 may be involved in the regulation of exercise-induced

\footnotetext{
* Correspondence to: Ikemoto, T., Functional Probe Research Laboratory, RIKEN Center for Molecular Imaging Science, Minatojima Minatomachi, Chuo-ku, Kobe, Hyogo 650-0047, Japan.

e-mail: tikemotoriko@riken.jp
}

GLUT4 translocation in skeletal muscles.

\section{MATERIALS AND METHODS}

Mice lacking sk-NSPL1: Sk-NSPL1-deificient mice were obtained from Lexicon Pharmaceuticals, Inc. (Wood Lands, TX, U.S.A.). PCR genotyping was used to distinguish the wild-type and targeted alleles as in a previous report [9]. All experiments used 8- to 12-week-old male mice after fasting for 15-17 hr. Animal experiments and care proceeded with the approval of our institutional Animal Care and Use Committee (Permission \#MAH18-04-6).

Samples from skeletal muscle: Three types of samples were used. First, whole skeletal muscle samples were freeze-dried and solubilized with $1 \%$ Triton X-100 in accordance with previous reports [11]. Second, F2 membrane fractions [3], which are abundant in sk-NSPL1 and GLUT4 as described previously [9], were prepared from the hindlimbs of mice after electrical stimulation, insulin injection, or AICAR administration. Third, we also used the membrane fraction, which was recovered at the $25 / 46 \%$ interfaces of discontinuous sucrose gradients $(25 / 46 \%$ membrane fraction), since sk-NSPL1 is also present in fractions other than the F2 membrane [9], without any stimulation.

Measurement of phosphate-metal affinity chromatography resin adsorbed protein: Whole skeletal muscle samples and F2 membrane fractions were solubilized or diluted with buffer A (Clontech, Mountain View, CA, U.S.A.), then incubated with phosphate-metal affinity chromatography resin (p-resin, Clontech). P-resin $(50 \mu l)$ was washed with 
distilled water and then washed by buffer A twice. Triton X100 -solubilized whole skeletal muscle $(6-10 \mathrm{mg} / \mathrm{m} l)$ with or without electrical stimulation was diluted with buffer A (1 $\mathrm{mg} / \mathrm{ml}, 100 \mu l)$ before mixing with p-resin. The F2 membrane fraction suspended in the final buffer $[10-20 \mathrm{mg} / \mathrm{ml}$; 0.3 M sucrose, 0.1 M KCl, $20 \mathrm{mM}$ PIPES/Tris, pH 7.0, protease inhibitor (Roche, Basel, Switzerland)] was also solubilized with buffer A $(1 \mathrm{mg} / \mathrm{ml}, 100 \mu l)$. The $25 / 46 \%$ membrane fraction was used to examine the change in skNSPL1 absorption with p-resin. The 25/46\% membrane fraction was incubated in a test solution $[6.67 \mathrm{mg} / \mathrm{ml}, 0.2 \mathrm{M}$ $\mathrm{NaCl}, 20 \mathrm{mM}$ PIPES/Tris, $\mathrm{pH}$ 7.0, $100 \mathrm{nM}$ thapsigargin, 2.5 $\mu \mathrm{M}$ calmodulin $(\mathrm{CaM})]$ various concentrations of $\mathrm{Ca}^{2+}$ buffered with $1 \mathrm{mM}$ EGTA in the presence or absence of ATP for $2 \mathrm{~min}$ to $18 \mathrm{hr}$ at $37^{\circ} \mathrm{C}$. Calculated total amounts $\mathrm{Ca}^{2+}$ added in test solution to make $0.1-10 \mu \mathrm{M}$ free $\mathrm{Ca}^{2+}$ with or without $3.14 \mathrm{mM}$ total ATP and $2.5 \mathrm{mM}$ total $\mathrm{Mg}^{2+}$ $\left(\mathrm{MgATP}^{-}=2.0 \mathrm{mM}\right.$ and free $\left.\mathrm{Mg}^{2+}=0.5 \mathrm{mM}\right)$ are as follows: $0.1 \mu \mathrm{M} \mathrm{Ca}^{2+}, 0.204 ; 1 \mu \mathrm{M} \mathrm{Ca}^{2+}, 0.720 ; 10 \mu \mathrm{M} \mathrm{Ca}^{2+}, 0.972$ (in $\mathrm{mM}$ ). At $100 \mu \mathrm{M} \mathrm{Ca}^{2+}$, EGTA was omitted from the test solution and $0.1 \mathrm{mM} \mathrm{CaCl}_{2}$ was added. After treatment with test solution, proteins from membrane fraction were solubilized with buffer A $(1 \mathrm{mg} / \mathrm{ml})$.

P-resin-adsorbed proteins after 30-min incubation of solubilized or diluted samples were washed 4 times with repetitive centrifugation and then eluted with SDS-sample buffer. Proteins eluted from $\mathrm{p}$-resin were estimated by western blotting analysis using specific antibodies, and a repeat of the experiment was conducted without $\mathrm{p}$-resin to analyze the total amount of each protein in the sample.

Antibodies: We obtained polyclonal antibodies from rabbit against synthetic peptides derived from the $\mathrm{C}$-terminus of mouse NSPL1 or from the N-terminus of mouse Nogo-C, which is a member of the reticulon family originated by Nogo gene [8]. Antibodies other than those for NSPL1 and Nogo-C were purchased from Cell Signaling Technology, Inc. (Danvers, MA, U.S.A.) or Affinity BioReagents (Rockford, IL, U.S.A.).

Separation of sk-NSPL1 with ion exchange resin: The 25/ $46 \%$ membrane fraction $(300 \mu \mathrm{g}, 10 \mathrm{mg} / \mathrm{m} l)$ was incubation for $2 \mathrm{~min}$ to $18 \mathrm{hr}$ in $100 \mu \mathrm{M} \mathrm{Ca}^{2+}$ at $37^{\circ} \mathrm{C}$ without ATP for protein dephosphorylation. Then, the sample was solubilized and diluted to a protein concentration of $1.0 \mathrm{mg} / \mathrm{m} l$ with $1 \%$ Triton X-100 in $20 \mathrm{mM}$ PIPES/Tris, $\mathrm{pH}$ 7.0. After centrifugation, the sample was loaded into $100 \mu \mathrm{l}$ of macroprep high $\mathrm{S}$ resin, and the unadsorbed flow-through was reloaded into the same volume of macro-prep high $\mathrm{Q}$ resin (Bio-rad, Hercules, CA, U.S.A.), which was pre-equilibrated with a solution $0.2 \%$ Triton $\mathrm{X}-100$. Protein in the resin was eluted with $100 \mu l$ of $1.25 \mathrm{M} \mathrm{NaCl}$. Eluted sample $(10 \mu l)$ was analyzed by western blotting.

Measurement of blood glucose in the whole mouse: The blood glucose level of anesthetized mice was measured with Glucocard-Diameter $\alpha$ (GT-1661; Arkray, Kyoto, Japan) as detailed in a previous report [9].

Data analysis: The data are presented as means \pm standard error of the mean (SEM). Paired data sets were tested using Student's paired $t$-test. Multiple comparisons were analyzed using one-way analysis of variance (ANOVA) for repeated measurements followed by Fisher's PLSD post hoc test. $P<0.05$ was considered statistically significant.

\section{RESULTS}

In sk-NSPL1-deficient muscles, exercise-induced glucose uptake is totally abolished with no change in insulininduced glucose uptake [9]. There are many reports showing that AMPK is activated and phosphorylated in relation with membrane translocation of GLUT4 under exercise [5, 13, 16]. In the present study, phosphorylated AMPK (pAMPK) was higher in muscle samples electrically stimulated (Fig. 1A). The increase of phosphorylated acetyl-CoA carboxylase (pACC) is consistent with the activation of AMPK under exercise (Fig. 1A) [5, 11]. However, the effect of AICAR on blood glucose levels was greatly diminished in sk-NSPL1-deficient mice (Fig. 1B vs. 1C), as was the case with electrical stimulation [9]. Thus, our results indicate that sk-NSPL1 could be involved downstream of the AMPK-mediated pathway in exercise-induced GLUT4 translocation.

Consistent with the results from phospho-protein specific antibodies, the amounts of eluted AMPK and ACC from presin were much larger in electrically stimulated samples (Fig. 2A, 2E-S). Moreover, a large amount of proline-rich Akt substrate of $40 \mathrm{kDa}$ protein (PRAS40), which is a protein related to the mammalian target of rapamycin (mTOR) signaling pathway [4], was eluted from p-resin in insulinstimulated muscle, which was consistent with detection by phospho-PRAS40 antibody (Fig. 2A, pPRAS40). Therefore, we concluded that p-resin can recognize the phosphorylated protein. We subsequently attempted to examine whether sk-NSPL1 itself was trapped by p-resin in response to electrical or insulin stimuli. When samples from whole skeletal muscle were loaded onto p-resin, sk-NSPL1 was not recognized in the elution (data not shown); however, the amount of sk-NSPL1 adsorbed by p-resin from the F2 membrane fraction from electrically stimulated muscle was greater than that from insulin stimulation or from resting muscle (Fig. 2B and 2D). In addition, AICAR administration also significantly enhanced both sk-NSPL1 and AMPK adsorption (Fig. 2C and 2D).

Next, we examined whether sk-NSPL1 in the isolated membrane fraction from resting muscle changed its adsorption onto p-resin in response to exogenous ATP. At $1 \mu \mathrm{M}$ $\mathrm{Ca}^{2+}$, the presence of ATP slightly enhanced sk-NSPL1 adsorption (Fig. 3A). Calmodulin (CaM), which regulates a multitude of enzymes in a $\mathrm{Ca}^{2+}$-dependent manner [1], further increased sk-NSPL1 adsorption (Fig. 3A, CaM). In the absence of ATP, sk-NSPL1 adsorption was gradually reduced as the $\mathrm{Ca}^{2+}$ in the test solution increased (Fig. 3B, - , and $3 \mathrm{C}$, closed circle). On the other hand, adsorbed skNSPL1 in the presence of ATP and CaM was potentiated in a $\mathrm{Ca}^{2+}$-dependent manner (Fig. 3B, +, and 3D, closed circle). For sk-NSPL1-deficient muscles, we found retention 
A

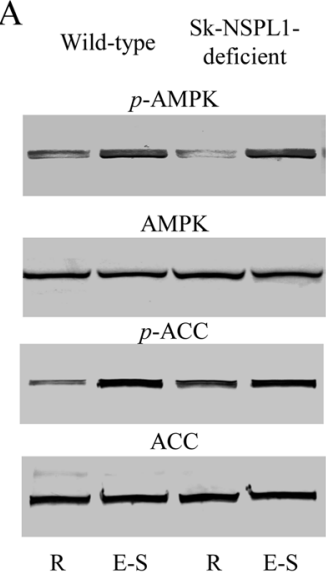

B

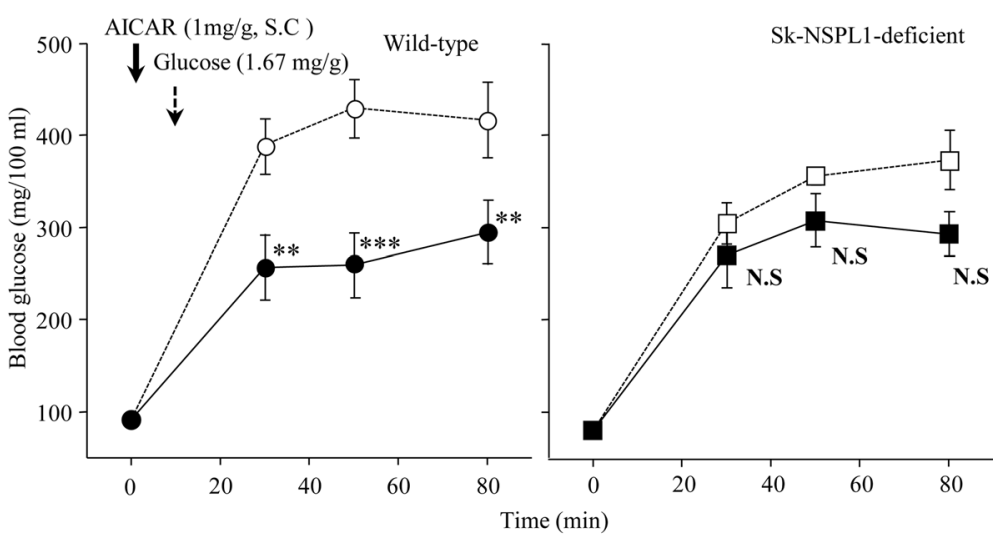

Fig. 1. Activation of AMPK and blood glucose levels in sk-NSPL1-deficient mice. A, Comparison of phosphorylation in AMPK and ACC. Solubilized protein $(45 \mu \mathrm{g})$ from freeze-dried skeletal muscle for $30-45$ min without any stimulation (Rest, R) and with electrical stimulation for $15 \mathrm{~min}$ (E-S) in wild-type and sk-NSPL1-deficient skeletal muscles were separated with SDS-PAGE and transferred onto PVDF membranes. Phospho-AMPK (p-AMPK), AMPK, phospho-ACC (p-ACC), and ACC were recognized by specific antibodies and AP-conjugated secondary antibodies. B and C, Changes in blood glucose concentrations were measured in wild-type (B) and sk-NSPL1deficient (C) mice under anesthesia. After measuring the fasting level of blood glucose concentrations at time $=0$, AICAR was instantly injected (s.c., bold arrow). After $10 \mathrm{~min}$, a high concentration of glucose was injected (s.c., dashed arrow). Control (open circles and open squares, high glucose injection alone), and AICAR (filled circles and filled squares). $\mathrm{n}=5$ in each experiment. ${ }^{*} * P<0.02, * * * P<0.01$ (against control values). N.S, not significant.

A

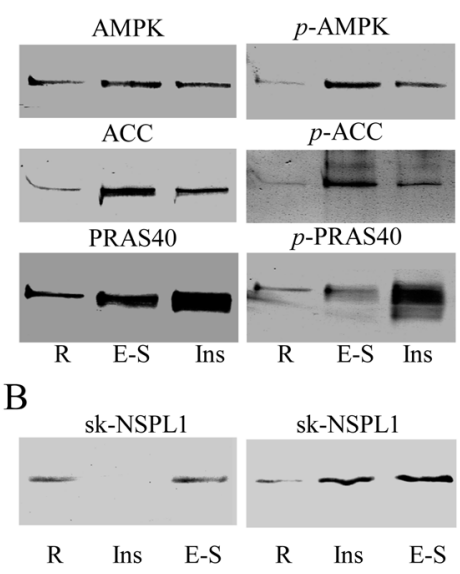

$\mathrm{C}$
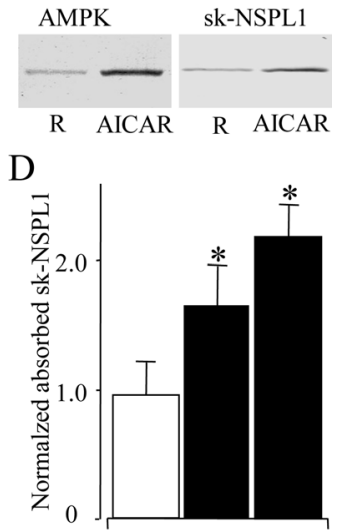

Ins E-S AICAR

Fig. 2. Sk-NSPL1 adsorption onto p-resin for skeletal muscles. A, Usefulness of p-resin to isolate phospho-protein from the solubilized F2 membrane fraction. Proteins eluted from p-resin were compared for the solubilized F2 membrane fraction without any stimulation (Rest, R) with electrical stimulation for 15 min (E-S) and with insulin (Ins, 2U) in wild-type using normal antibodies (AMPK, ACC PRAS40) and antibodies for their phosphorylated forms (pAMPK, pACC, pPRAS40). B, Two typical recordings of sk-NSPL1 in each sample. Sk-NSPL1 adsorption from some samples was enhanced with insulin, but for others was reduced by it (Ins). C, AICAR-induced enhancement of AMPK and sk-NSPL1 adsorption. After administration of AICAR $(1 \mathrm{mg} / \mathrm{g})$ for $45 \mathrm{~min}$, the isolated F2 membrane fraction from muscle was solubilized and mixed with p-resin. D, Comparison of sk-NSPL1 adsorption from multiple preparations. Electrical stimulation $(n=5)$ and AICAR administration $(n=3)$, but without insulin $(n=5)$, significantly increased both AMPK and sk-NSPL1 adsorption. $* P<0.05$.

in p-resin of Nogo-C, which is a member of the reticulon family with the same molecular weight as sk-NSPL1 and is expressed in skeletal muscle [8]. However, no $\mathrm{Ca}^{2+}$-dependent change of Nogo-C was observed in the absence or presence of ATP (Fig. 3B-D). In addition to Nogo-C, no significant degradations were observed in a selection of proteins [calsequestrin (CSQ), PRAS40, sk-NSPL1] from normal muscles after a 2-min incubation in test solution (Fig. 3E). The data in Fig. 3B show that the amount of loaded skNSPL1 onto p-resin was not changed in the test solution. Therefore, these data suggest that trapped sk-NSPL1 in presin is reduced in the absence of ATP and increased in the presence of ATP.

Since CaM enhanced the effect of $\mathrm{Ca}^{2+}$ on sk-NSPL1 adsorption to p-resin, we suspected that $\mathrm{Ca}^{2+}-\mathrm{CaM}$ dependent protein kinase was involved. We thus tested the effect of KN-62 [16] and ML-7 [2], inhibitors of $\mathrm{Ca}^{2+}$-CaM dependent protein kinase. KN-62, but not ML-7 inhibited the increase of sk-NSPL1 adsorption (Fig. 4A). We also tested the effect of phosphatase inhibitors. A phosphatase inhibitor cocktail (dephostatin, cypermethrin, and Okadaic Acid; Merck, Darmstadt, Land Hessen, Germany) inhibited the decrease of sk-NSPL1 adsorption in the absence of ATP (Fig. 4B).

Dantrolene (Dan) is an inhibitor of exercise-induced GLUT4 translocation, and sk-NSPL1 is a dantrolene receptor [9]. However, Dan showed no effect on sk-NSPL1 adsorption (Fig. 4A).

Finally, we examined the difference in charge of the protein between p-resin adsorbed and unabsorbed sk-NSPL1 using ion-exchange resin, since phosphorylation may impart 
A

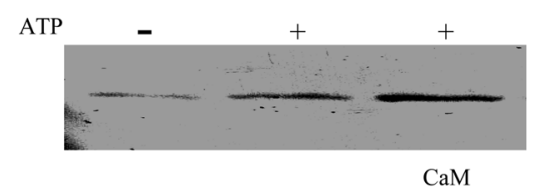

B
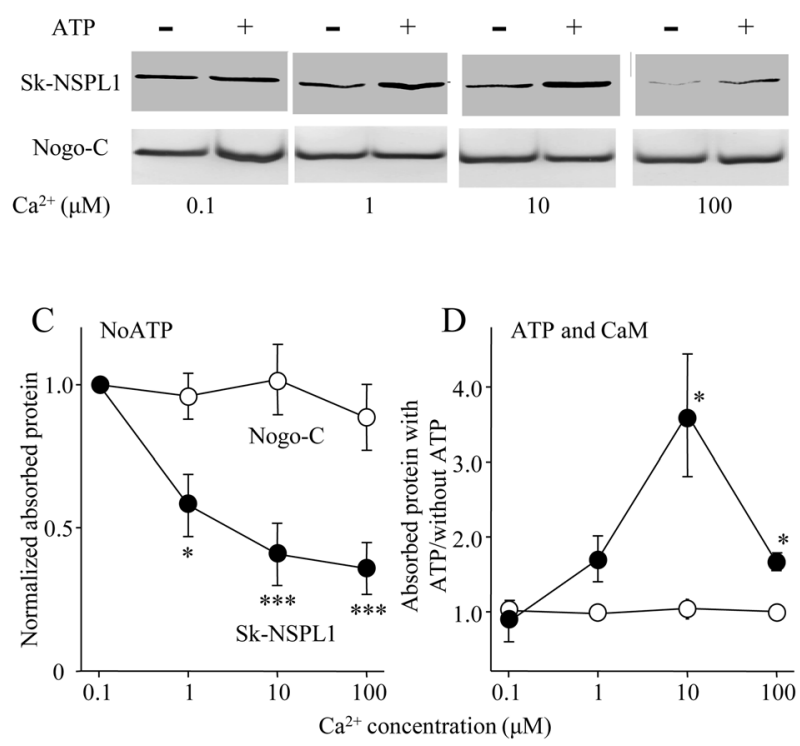

E

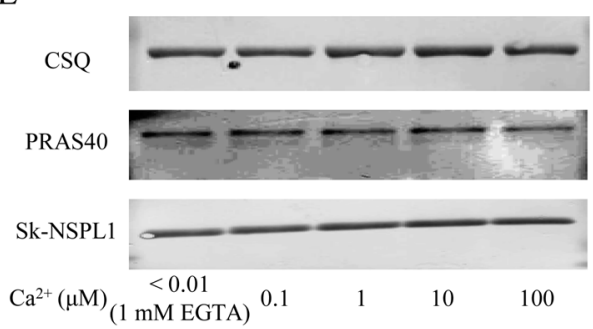

Fig. 3. $\mathrm{Ca}^{2+}$-dependent enhancement of isolated sk-NSPL1 adsorption. A, Increase of sk-NSPL1 adsorption in p-resin after treatment of ATP with CaM. At $1 \mu \mathrm{M} \mathrm{Ca}^{2+}$ concentration, the sk-NSPL1-containing 25/46\% membrane fraction was incubated in the absence $(-)$ or presence of ATP $(+)$ with or without CaM $(2.5 \mu \mathrm{M})$ for $2 \mathrm{~min}$. B-D, Effect of various concentrations of $\mathrm{Ca}^{2+}$ on sk-NSPL1 (filled circles) and Nogo-C (open circles) adsorption. After treatment with $0.1-100 \mu \mathrm{M} \mathrm{Ca}{ }^{2+}$ in the absence $(\mathrm{B},-; \mathrm{C})$ or presence of ATP with $\mathrm{CaM}(\mathrm{B},+; \mathrm{D})$, each adsorbed protein in p-resin was normalized to its levels at 0.1 $\mu \mathrm{M} \mathrm{Ca}{ }^{2+}$ in the absence of ATP (C), and the ratio of protein adsorption with ATP or without ATP (D) was plotted against $\mathrm{Ca}^{2+}$ concentration $(\mathrm{n}=4-9) . \quad * P<0.05, * * * P<0.01$ (against Nogo-C). E, Typical results of protein degradation at $0-100 \mu \mathrm{M}$ $\mathrm{Ca}^{2+}$. The $25 / 46 \%$ membrane fraction from wild-type muscles was incubated with test solution with $100 \mu \mathrm{M} \mathrm{Ca}^{2+}$ for $2 \mathrm{~min}$. Then the samples were solubilized with buffer A and centrifuged. Changes of calsequestrin (CSQ), PRAS40, and sk-NSPL1 in the membrane fraction were analyzed by western blotting.
A
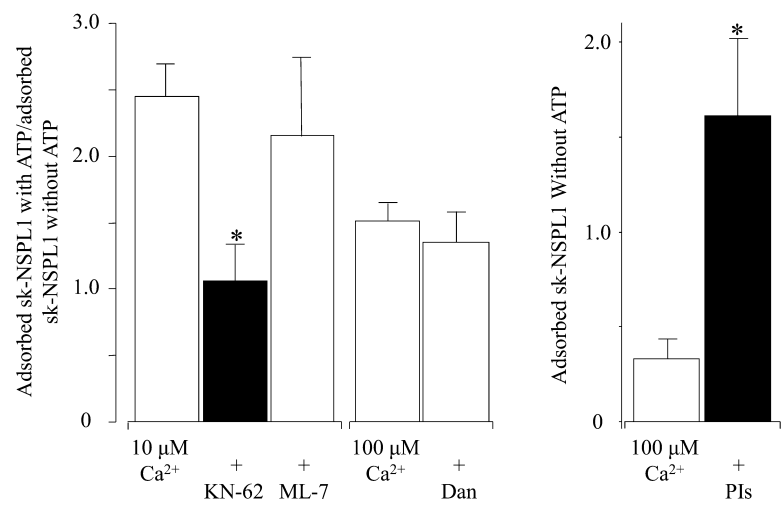

Fig. 4. Effects of phosphorylation related compounds and dantrolene on sk-NSPL1 adsorption. After treatment with KN-62 (10 $\mu \mathrm{M})$ or ML-7 $(3 \mu \mathrm{M})$ in a test solution containing 10 or $100 \mu \mathrm{M}$ $\mathrm{Ca}^{2+}$ with ATP and CaM, sk-NSPL1 adsorption was compared with that from a control experiment $(A, n=4$ for kinase inhibitors and $\mathrm{n}=5$ for Dan, $25 \mu \mathrm{M})$. In the absence of ATP and CaM, phosphatase inhibitors (dephostin, $20 \mu \mathrm{M}$; okadaic acid, $1 \mathrm{nM}$; and cypermethrin, $20 \mathrm{nM}$; PIs) in test solution at $100 \mu \mathrm{M} \mathrm{Ca}^{2+}$ were compared with control values $(B, n=9)$.

a negative charge on the targeted protein. Long-term incubation in test solution with high $\mathrm{Ca}^{2+}(100 \mu \mathrm{M})$ fully abolished p-resin adsorption of sk-NSPL1 (Fig. 5A, 2 min vs. $3-$ $18 \mathrm{hr}$ ), although it was not fully digested under the same conditions (Fig. 5A vs. 5B, 3-6 hr). After incubation of skNSPL1 in the $25 / 46 \%$ membrane fraction under the same conditions, anion-exchange resin-trapped sk-NSPL1 was drastically reduced (Fig. 5C, 3 and $6 \mathrm{hr}$, Q) compared to cation-exchange resin-trapped (S) sk-NSPL1. Thus, a change in the charge of sk-NSPL1 may be induced by contraction of skeletal muscle.

\section{DISCUSSION}

In this study, we demonstrated that contraction-stimulated pAMPK, pACC, and insulin-stimulated pPRAS40 from skeletal muscles were adsorbed efficiently by PMAC resin (p-resin). In addition, p-resin adsorbed sk-NSPL1 from electrically or AICAR-stimulated skeletal muscle to a greater degree than that from control muscles (Fig. 2).

The amount of sk-NSPL1 adsorption from muscle without stimuli was significantly affected by the presence of physiological levels of $\mathrm{CaM}, \mathrm{Ca}^{2+}$, and ATP, although that of Nogo-C was unaffected (Fig. 3). Furthermore, treatment of the membrane fraction with KN-62 and phosphatase inhibitors changed sk-NSPL1 adsorption onto p-resin (Fig. 4A and 4B). These results raise two possibilities: one is that sk-NSPL1 is directly phosphorylated by a certain kinase(s) that may cause a functional change in sk-NSPL1 itself, and the other is that phosphorylation of an sk-NSPL1-associated protein may cause a change in sk-NSPL1, although neither phenomenon has been reported yet. It must be noted that Nogo-C adsorption was derived from properties other than 

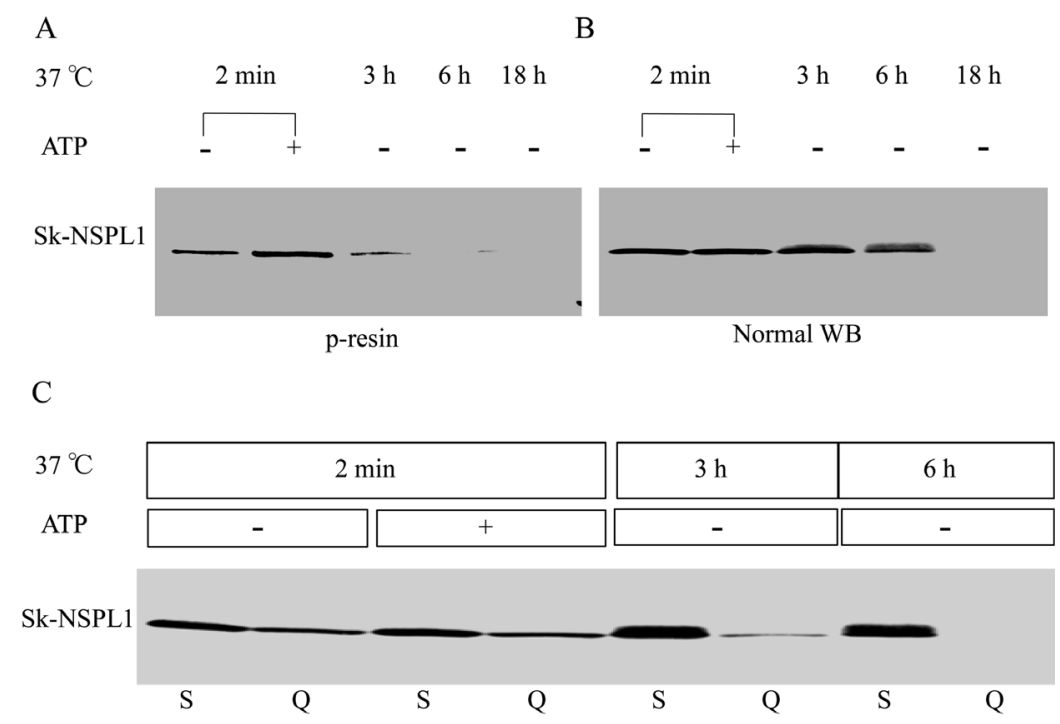

Fig. 5. Conditions on sk-NSPL1 adsorption with p-resin. A and B, After long-term incubation in test solution with $(+)$ or without ATP and $\mathrm{CaM}$ at $100 \mu \mathrm{M} \mathrm{Ca}^{2+}$, skNSPL1 adsorption from the $25 / 46 \%$ membrane fraction $(100 \mu \mathrm{g})$ with p-resin (A) was compared with the degree of degradation in sk-NSPL1 in the membrane fraction (1 $\mu \mathrm{g})$ without $\mathrm{p}$-resin $(\mathrm{B}$, normal WB). C, Larger amounts of sample $(300 \mu \mathrm{g})$ were incubated in the same test solution, and then after solubilization and dilution $(\times 10)$ to reduce the ionic concentration, samples were loaded onto high S-resin twice. Flowthrough from high $\mathrm{S}$ resin was reloaded onto the high Q resin. Protein adsorbed to the resin was eluted by $1.25 \mathrm{M} \mathrm{NaCl}$ and analyzed by western blotting.

phosphorylation, since its adsorption in p-resin was not affected by the presence or absence of ATP (Fig. 3B-D).

In test solution, $1-100 \mu \mathrm{M} \mathrm{Ca}^{2+}$, which is comparable to $\left[\mathrm{Ca}^{2+}\right]_{\mathrm{i}}$ for activating the contractile system of skeletal muscle, increased sk-NSPL1 adsorption, suggesting that skNSPL1 might function in the elevation of $[\mathrm{Ca}]_{\mathrm{i}}$ under exercise (Fig. 3).

$\mathrm{Ca}^{2+}-\mathrm{CaM}$ dependent kinase II, which is activated in exercise-induced GLUT4 translocation [15, 16], might phosphorylate sk-NSPL1 or an associated protein judging from the inhibitory effect of KN-62 on sk-NSPL1 adsorption in p-resin (Fig. 4A), although other types of kinase might be involved. In particular, it must be determined whether AMPK can modulate the adsorption of isolated skNSPL1, as was the case with in situ sk-NSPL1 (Fig. 2).

Both pAMPK and pACC were increased by exercise in sk-NSPL1-deficient muscles; however, glucose metabolism in the whole mouse body was significantly changed (Fig. 1). This indicates that sk-NSPL1 regulated the exerciseinduced GLUT4 translocation further downstream of AMPK-mediated signal transduction. Although there is no doubt that AMP is a major regulating factor in GLUT4 translocation in skeletal muscles, the current focus is on determining the target protein(s) for AMPK [5] and contraction-related kinase, for example CaMKII [15]. The results in this report suggest that sk-NSPL1 may be a candidate.

The most important result of this study was a change in charge of sk-NSPL1 under dephosphorylating conditions, which may be a quite different from that under phosphorylating conditions (Fig. 5A and 5B). The isoelectric point of sk-NSPL1 is calculated to be about 10 from its primary amino acid sequence [6]. This means that sk-NSPL1 is a basic protein and that a cation exchanger might strongly capture it at neutral $\mathrm{pH}$. However, a large amount of solubilized sk-NSPL1 was adsorbed on an anion exchanger (High Q) without adsorption on a cation exchanger (High S). This reaction was promoted after incubation of sk-NSPL1 with ATP (Fig. 5A). On the other hand, long-term incubation in the absence of ATP revealed sk-NSPL1 adsorption only on the cationic exchanger (Fig. 5C). This suggests that a phosphorylation-mediated pathway may cause a drastic change in the function of sk-NSPL1.

Dan did not inhibit sk-NSPL1 adsorption (Fig. 4A). Thus, Dan influences the function of sk-NSPL1 after the change in phosphorylation; for example, inhibition of binding activity with another factor that might be a key protein in GLUT4 translocation. To fully understand exerciseinduced GLUT 4 translocation, it is necessary to discover the targeted or associated protein(s) of sk-NSPL1 after phosphorylation or dephosphorylation in skeletal muscle.

ACKNOWLEDGMENT. This work was supported by the molecular imaging program of "Research Base for Exploring New Drugs", from the Ministry of Education, Culture, Sports, Science and Technology (MEXT), Japan. 


\section{REFERENCES}

1. Cheung, W. Y. 1980. Calmodulin plays a pivotal role in cellular regulation. Science 207: 19-27.

2. Dhawan, J. and Helfman, D. M. 2004. Modulation of actomyosin contractility in skeletal muscle myoblasts uncouples growth arrest from differentiation. J. Cell. Sci. 117: 37353748.

3. Dhawan, J. and Helfman, D. M. 2004. Modulation of actomyosin contractility in skeletal muscle myoblasts uncouples growth arrest from differentiation. J. Cell. Sci. 117: 37353748.

4. Dunlop, E. A. and Tee, A. R. 2009. Mammalian target of rapamycin complex 1: Signaling inputs, substrates and feedback mechanisms. Cell. Signal. 21: 827-835.

5. Funai. K. and Cartee, G. D. 2009. Inhibition of contractionstimulated AMP-activated Protein kinase inhibits contractionstimulated increases in PAS-TBC1D1 and glucose transport without altering PAS-AS160 in rat skeletal muscle. Diabetes 58: 1096-1104.

6. Geisler, J. G., Stubbs, L. J., Wasserman, W. W. and Mucenski, M. L. 1998. Molecular cloning of a novel mouse gene with predominant muscle and neural expression. Mamm. Genome 9: 274-282.

7. Huang, S. and Czech, M. P. 2007. The GLUT4 glucose transporter. Cell Metab. 5: 237-252.

8. Huber, A. B., Weinmann, O., Brösamle, C., Oertle, T. and Schwab, M. E. 2002. Patterns of Nogo mRNA and protein expression in the developing and adult rat and after CNS lesions. J. Neurosci. 22: 3553-3567.

9. Ikemoto, T., Hosoya, T., Takata, K., Aoyama, H., Hiramatsu, T., Onoe, H., Suzuki, M. and Endo, M. 2009. Functional role of neuroendocrine-specific protein-like 1 in membrane translocation of GLUT4. Diabetes 58: 2802-2812.
10. Kånge, R., Seldit, U., Granberg, M., Lindberg, U., Ekstrand, G., Ek, B. and Gustafsson, M. 2005. Comparison of different IMAC techniques used for enrichment of phosphorylated Peptides. J. Biomol. Tech. 16: 91-103.

11. McConell, G. K., Manimmanakorn, A., Lee-Young, R. S., Kemp, B. E., Linden, K. C. and Wadley, G. D. 2008. Differential attenuation of AMPK activation during acute exercise following exercise training or AICAR treatment. J. Appl. Physiol. 105: 1422-1427.

12. Merrill, G. F., Kurth, E. J., Hardie, D. G. and Winder, W. W. 1997. AICA riboside increases AMP-activated protein kinase, fatty acid oxidation, and glucose uptake in rat muscle. Am. $J$. Physiol. Endocrinol. Metab. 273: E1107-1112.

13. Mu, J., Brozinick, Jr. J. T., Valladares, O., Bucan, M. and Birnbaum, M. J. 2001. A role for AMP-activated protein kinase in contraction- and hypoxia-regulated glucose transport in skeletal muscle. Mol. Cell 7: 1085-1094.

14. Röckl, K. S., Witczk, C. A. and Goodyear, L. J. 2008. Signaling mechanisms in skeletal muscle: acute responses and chronic adaptations to exercise. IUBMB Life 60: 145-153.

15. Witczak, C. A., Jessen, N., Warro, D. M., Toyoda, T., Fujii, N., Anderson M. E., Hirshman M. F. and Goodyear, L. J. 2010. CaMKII regulates contraction- but not insulin-induced glucose uptake in mouse skeletal muscle. Am. J. Physiol. Endocrinol. Metab. 298: E1150-1160.

16. Wright, D. C., Hucker, K. A., Holloszy, J. O. and Han, D. H. 2004. $\mathrm{Ca}^{2+}$ and AMPK both mediate stimulation of glucose transport by muscle contractions. Diabetes 53: 330-335.

17. Zheng, D., Perianayagam, A., Lee, D. H., Brannan, M. D., Yang, L. E., Tellalian, D., Chen, P., Lemieux, K., Marette, A., Youn, J. H. and McDonough, A. A. 2008. AMPK activation with AICAR provokes an acute fall in plasma $\left[\mathrm{K}^{+}\right] . A m . J$. Physiol. Cell. Physiol. 294: C126-135. 